\title{
Effect of Hydrotubation in Unexplained Infertility - A Randomized Controlled Trial
}

\author{
Shazia Saaqib, ${ }^{1}$ Muhammad Arshad Chohan, ${ }^{2}$ Misbah Ashraf, ${ }^{3}$ Ammara Mumtaz ${ }^{4}$
}

\begin{abstract}
Unexplained infertility is a major gynecological problem with complex etiolgy. Partial tubal blockage with inflammatory reaction and thick mucus plugs in fallopian tubes and cervix is one of the likely causes in such cases. This study is based on the hypothesis that women having unexplained infertility could be helped by transcervical flushing of fluid through the fallopian tubes which would remove reversible blocking factors and help in achieving pregnancy.
\end{abstract}

Objective: To compare the outcome of hydrotubation in unexplained infertility in clomiphene citrate stimulated cycles with control group (no Hydrotubation).

Setting: Infertility Centre of a Public Sector Hospital.

Study Design: A two years Prospective randomized controlled trial from Jan 2014 to Dec 2015.

Method: 128 women participated in the study. Parti-

\footnotetext{
${ }^{1}$ Senior Registrar, Dept of Obstetrics \& Gynaecology

Lady Willingdon Hospital, Lahore

${ }^{2}$ Professor of Obstetrics \& Gynaecology, KEMU / Lady Willingdon Hospital, Lahore

${ }^{3}$ PG Trainee Dept of Obstetrics \& Gynaecology

Lady Willingdon Hospital, Lahore

${ }^{4}$ PG Trainee Dept of Obstetrics \& Gynaecology

Lady Willingdon Hospital, Lahore
}

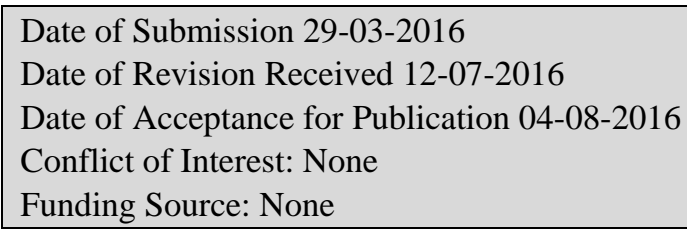

\section{Contribution}

All Authors have contributed in Study Design, Data Collection, Data Analysis, Data Interpretation, Manuscript Writing and Approval. cipants were divided into study and control group with 64 members in each group. Ovulation induction was done with clomiphene citrate in all the participants and confirmed by ovulation detection strips and follicular tracking on Ultrasonography. 50 cc cocktail of injection (inj.) kanamycin $1 \mathrm{gm}$, inj. hydrocortisone and normal saline was injected transcervically in study group for three consecutive days from the next day after end of menstruation. No hydrotubation was done in control group. Main outcome measures were number of conceptions within next 3 cycles and complications of hydrotubation.

Results: In hydrotubation group, 20 women conceived. Out of these, 14 women conceived in the same cycle in which hydrotubation was done while 6 pregnancies occurred in subsequent cycle. 4 pregnancies occurred in the control group. Complications of hydrotubation were mild abdominal pain (59 women), vomiting (49 women) and Per vaginum bleeding (18 women). There were significantly more pregnancies in women who experienced right shoulder tip pain after the procedure.

Conclusion: In women with unexplained infertility, tubal hydration can increase conception rate and decrease the time to achieve pregnancy.

Key words: Infertility, unexplained, hydrotubation, conception, vomiting, pain.

\section{Introduction}

Infertility is a major gynecological problem with complex etiology and about $10 \%$ cases remain unexplained after investigation. ${ }^{1}$ These investigations include husband's semen analysis, hysterosalpingograghy for tubal patency, postcoital cervical mucus testing for hostile cervical mucus and detection of ovulation. Ovulation can be detected by history of menstrual cycles regu- 
larity, hormonal profile of serum follicle stimulating hormone, serum leutinizing hormone, serum prolactin levels and thyroid function tests. Ovulation can further be confirmed by ovulation detecting strips. A couple is said to have unexplained infertility if all the investigations are within normal limits. ${ }^{2}$

The treatment of unexplained infertility is empirical, and many different regimens have been tried.-5 The first line approach to treat unexplained infertility is to stimulate oocyte production by ovarian hyperstimulation with clomiphene citrate. Its administration is associated with multifollicular development in most cases, which may result in enhanced fertility. ${ }^{4,5}$

Tubal disease is considered to be the major underlying cause in unexplained infertility even if the tubes are patent on radiological testing ${ }^{2}$. It is classified as mild, moderate and severe depending on tubal patency, morphology, fimbrial structure and adhesions. ${ }^{6}$ Significantly improved conception rate has been achieved by hydrotubation in unexplained infertility. ${ }^{5-7}$

Hydrotubation is Trans cervical flushing of fluid through the fallopian tubes. This technique has been used to test the patency of fallopian tubes and has been found to improve conception rate in infertile couples for about 50 years. ${ }^{8}$ Patency of tubes is confirmed by lack of resistance to the passage of fluid through the cervix and right shoulder tip pain. This technique is cost effective, cheap, easy to perform and does not require special arrangements like theatre facilities. It is now being used as a therapeutic measure of unexplained infertility in Pakistan ${ }^{1}$ and abroad. ${ }^{2-5}$ The therapeutic effect of hydrotubation on infertility can either be mechanical or immunological. ${ }^{2,7,9}$ It can act by dislodgment of debris in fallopian tubes or by separating the loose adhesions of the tubes. It can also remove thick hostile cervical mucus which may hinder the passage of sperms through the cervix. ${ }^{9}$ Antibiotics and steroid injections in flushing media can reduce inflammatory reaction and swelling in tubes. There may be stimulation of peristalsis by flushing of fluid, inhibition of phagocytosis of sperms by macrophages and dilution of toxic factors such as cytokines in endometriosis. $^{7}$ The adverse effects of hydrotubation could be ectopic pregnancy, increased risk of infection, sever pain, nausea, vomiting and peritonitis. ${ }^{10-12}$

This study is based on the hypothesis that women having unexplained infertility could be helped by hydrotubation which would remove reversible blocking factors and hostile elements and result in achieving pregnancy.
The study was designed to evaluate the effect of hydrotubation in treatment of unexplained infertility in clomiphene citrate stimulated cycles by comparing the outcome of hydrotubation versus no hydrotubation (in terms of number of conceptions achieved and complications of hydrotubation).

\section{Patients and Methods}

This study was conducted at infertility Centre of a Public Sector Hospital for 2 years (from Jan 2014 to Dec 2015) after approval of research protocol by ethical committee of King Edward Medical University. It was a prospective randomized controlled trial. A total of 960 women attended infertility clinic during this period. Their age, parity, duration of infertility, previous history of menstrual cycles, abdominal and gynecological surgeries, miscarriages, and medical disorders were noted. Baseline investigations like blood group and hemoglobin percentage, hepatitis screening, hormone profile, ultrasonography, husband semen analysis and hysterosalpingography were planned at first visit to infertility clinic. Inclusion criteria of the study were age up to 40 years with primary or secondary Infertility of 2 years or more, regular menstrual cycles and all investigations within normal limits with dye spillage on hysterosalpingograghy. Exclusion criteria were complete tubal blockage, male factor infertility, active pelvic infection and major medical illness like uncontrolled diabetes, hypertension and hepatitis, renal or cardiac disease. Women who fulfilled the criteria were counselled about empirical nature of procedure and possible complications. 128 women were included in the study based (calculation on total sample size at statistical power of $0.8, \mathrm{p}$ value of 0.05 and anticipated effect size of 0.5 ) after consent to participate in the study. They were randomly divided into study and control group with 64 participants per group according to simple random number software. The participants of both groups were treated by ovulation induction with clomiphene citrate $100 \mathrm{mg}$ twice daily from day $2-6$ of the menstrual cycle. In study group, ovulation induction was followed by hydrotubation done for 3 consecutive days after periods. The method consisted of passing Foleys catheter through the cervix and inflating balloon with $3 \mathrm{cc}$ distilled water. A cocktail was made in $50 \mathrm{cc}$ bladder wash syringe of injection hydrocortisone $1 \mathrm{gm}$, in kanamycin $1 \mathrm{gm}$ after testing hypersensitivity and normal saline up to $50 \mathrm{cc}$, injected transcervically into the genital tract. Post proce- 
dure complications were recorded and patients were sent home on analgesic (acetaminophen) and antibiotic (erythrocin and metronidazole) for 5 days. The ovulation induction was not followed by hydrotubationin control group. Ovulation was confirmed by ovulation detection strips and follicular tracking on USG in both groups. Urine test for pregnancy was done if woman missed her periods and transabdominal scan was performed to confirm fetal cardiac activity and intrauterine pregnancy. Both the groups were compared for their outcome in unexplained infertility.

Main outcome measures were number of conceptions within next 3 cycles and complications of hydrotubation.

Variables analyzed on SPSS 20 package. The numerical variables were calculated as mean \pm SD and difference between groups was compared by independent $t$ test $(\mathrm{P}<0.05)$. Chi-square test $\left(\mathrm{X}_{2}\right)$ was used to compare the nominal data. Spearman's test was used to establish correlation between relevant parameters.

\section{Results}

128 women were included in the study with 64 women each in study and control group. Demographic features of participants that could influence results of study were age, duration of infertility, type of infertility, previous surgeries and miscarriages (Table 1).

Mean age was 30.25 years in study group and 30.678 years in control group with wide range of 24 to 40 years. There were more participants with secondary infertility as compared to primary infertility in both

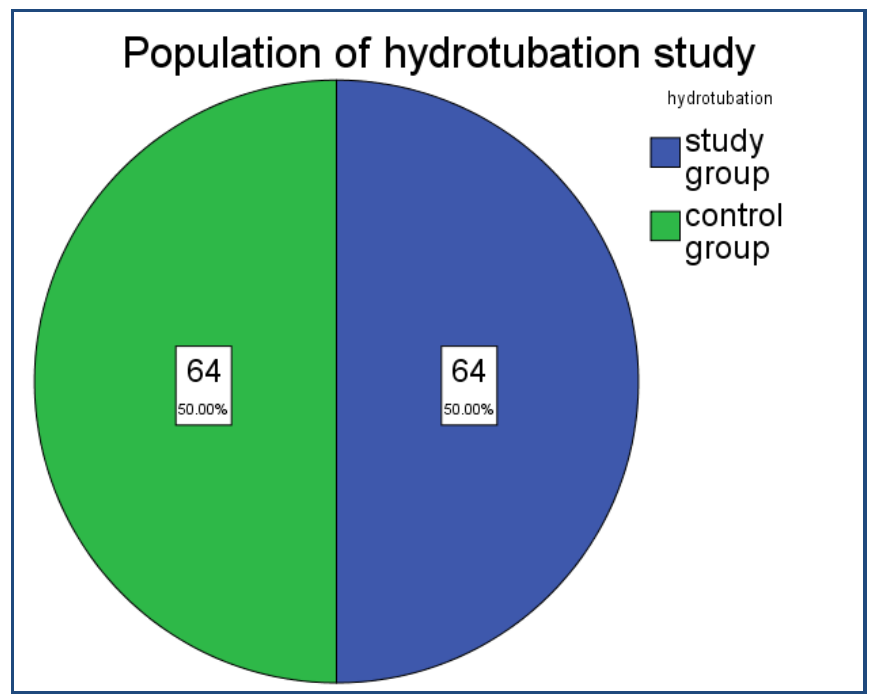

Fig. 1: Distribution of population in study and control group.

groups. Women with history of previous surgeries and miscarriages were also included provided there was dye spill on hysterosalpingography (Table 2). Statistically there were no significant differences in demographic features and criteria of both groups which could influence the results of study.

Complications like lower abdominal pain (59), vomiting (49), per vaginum bleeding and right shoulder tip pain were experienced in a large number of cases but these minor problems were overcome with reassurance, analgesic and antiemetic. There was not a single case of life threatening complications like vasovagal shock or ectopic pregnancy.

Table 1:

Demographic

features of study

population.

\begin{tabular}{|l|lr|rr|l|l|}
\hline Demographic Features & \multicolumn{2}{|c|}{$\begin{array}{c}\text { Study Group }(\mathbf{N}=\mathbf{6 4}) \\
\text { Mean } \pm \text { SD Deviation S.E. }\end{array}$} & \multicolumn{2}{|c|}{$\begin{array}{c}\text { Control group }(\mathbf{N}=64) \\
\text { Mean } \pm \text { SD S.E. }\end{array}$} & $\begin{array}{l}\text { P } \\
\text { value }\end{array}$ \\
\hline Age (years) Range $24-40$ & $30.25 \pm 3.647$ & 0.456 & $30.687 \pm 3.758$ & 0.470 & 0.505 \\
\hline Duration of infertility & $4.078 \pm 1.863$ & 0.232 & $4.625 \pm 1.714$ & 0.214 & 0.086 \\
\hline
\end{tabular}

Table 2: Characteristic of study population.

\begin{tabular}{|l|l|c|c|c|c|}
\hline \multicolumn{2}{|l|}{ Characteristics } & $\begin{array}{c}\text { Study } \\
\text { Group (n) }\end{array}$ & $\begin{array}{c}\text { Control } \\
\text { Group (n) }\end{array}$ & $\begin{array}{c}\text { Chi-Square } \\
\text { Test }\end{array}$ & P value \\
\hline \multirow{2}{*}{$\begin{array}{l}\text { Type of } \\
\text { infertility }\end{array}$} & Primary & 23 & 24 & \multirow{2}{*}{0.034} & 0.855 \\
\cline { 2 - 4 } & Secondary & 41 & 40 & & 0.723 \\
\hline Pervious surgeries & 35 & 33 & 0.125 & 0.725 \\
\hline Previous Miscarriages & 34 & 37 & 0.285 & 0.594 \\
\hline
\end{tabular}




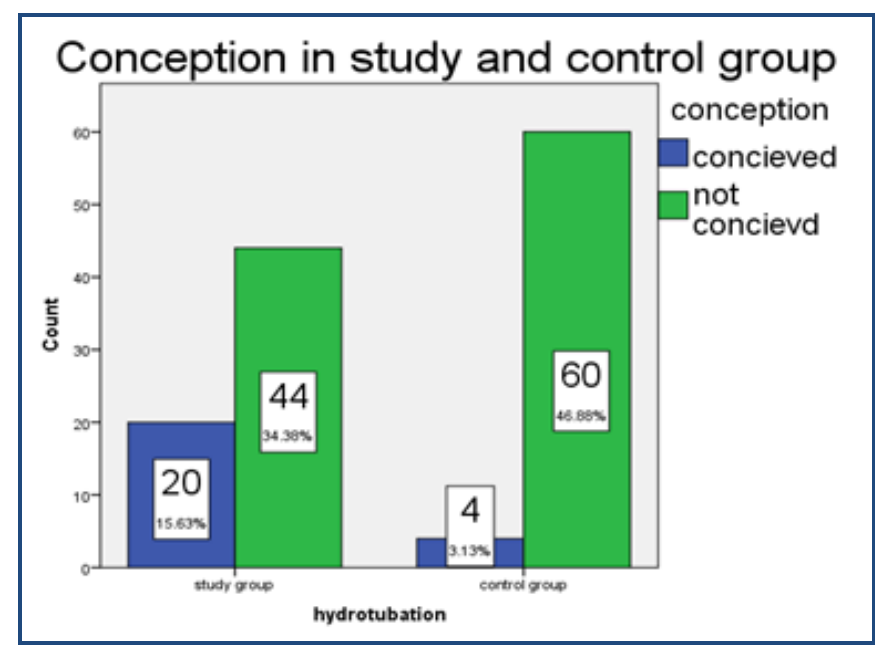

(Chi square 13.128 and $\mathrm{p}<0.05$ )

Fig. 2: Comparison of Conception rate in study group and control group.

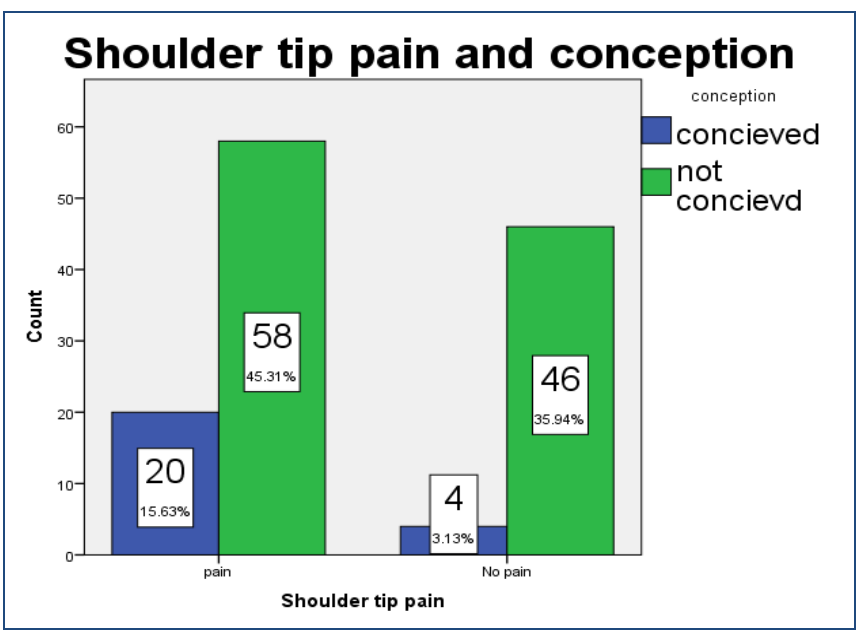

Fig. 3: Shoulder tip pain and conception in hydrotubation group and control group.

Table 3: Correlation of study parameters with conception.

\begin{tabular}{|l|c|c|}
\hline \multicolumn{1}{|c|}{ Results } & $\begin{array}{c}\text { Correlation } \\
\text { with } \\
\text { Conception }\end{array}$ & $\begin{array}{c}\text { Spearman Rho } \\
\text { Correlation } \\
\text { Coefficient }\end{array}$ \\
\hline Hydrotubation & +ive & 0.320 \\
\hline Right shoulder tip pain & +ive & 0.224 \\
\hline
\end{tabular}

Hydrotubation was found to be effective to improve fertility in unexplained cases and right shoulder tip pain was an effective parameter statistically to predict pregnancy (Table 3 ).
Majority of the patients conceived in the same cycle in which hydrotubation was done. Number of conceptions decreased in the next cycle and no conception occurred in the third cycle. This difference proved to be highly significant with chi-square test.

\section{Discussion}

The success of hydrotubation in different studies carried out indifferent geographical locations greatly varies. $^{2-5,13}$ The local data for comparison of success rate is not available but hydrotubation has been carried out and mentioned in a local descriptive study. ${ }^{1}$ The increased conception rate with this procedure has been proven in this study and in previous studies. ${ }^{3-5,7}$ In a prospective randomized trial of hydrotubation versus no hydrotubation before intrauterine insemination, results are not statistically significant yet the conception rate is more in hydrotubation group. ${ }^{11}$ This differrence in results is likely to be due to difference in demographic features of the study population, the media used for flushing and skills of the surgeon. The better prognosis of this study could be due to carefully selected healthy population with majority of women in younger age group. Advice about most fertile period of the cycle and confirmation of ovulation also increased the fecundity of study group as the couple knew the right time of conception. The higher success rate may also be due to use of steroid and antibiotic which have got anti-inflammatory properties. These drugs can reduce edematous changes and excessive thick mucopurulent fluid in the tube which might have been responsible for infertility. The sample size of this study is small to prove this effect but this observation is favoring the therapeutic effect of the medicines used.

The success rate is lower in studies where lesser amount of fluid is used for hydrotubation. ${ }^{8}$ The hydrostatic effect of the amount of fluid used can remove the obstructive elements in the tube and washing of tube with normal saline can dilute toxic and inflammatory factors. In this study, 50cc fluid was used daily to wash the tubes for 3days. There was a slight increase in discomfort of the patient in terms of lower abdominal pain, nausea and vomiting and right shoulder tip pain but greater chance of achieving the pregnancy makes even these complications agreeable to the womens compared to unsuccessful treatment with lesser fluid volume injected and lesser pain experienced. The use of Lidocaine in the above mentioned study results in non significant increase in pregnancy rate without significant difference in pain intensity. ${ }^{10}$ 
In another study with a much larger study population, success rate is much higher (75\%)as compared to this $\operatorname{study}(25 \%){ }^{7}$ Inclusion of tubal factor infertility in that study may be the reason for difference in results. Hydrotubation has proved to be more effective in populations with mild tubal disease as compared to moderate and sever tubal factor infertility.

The studies of infertility carried out after deciding the causative factor have a higher success rate where cervical factor is the causative agent and aspiration of cervical mucus improves fertility. ${ }^{9}$ In unexplained infertility, removal of hostile cervical mucus and flushing out the inspissated mucous plugs in the cervix and in fallopian tubes may be one of the mechanisms of action of hydrotubation. This observation has also been made in another study in which therapeutic effect of diagnostic test i.e. Hysterosalpingo - contrast sonography (carried out like hydrotubation but with contrast medium) has a higher conception rate $(22 \%){ }^{8}$ The pregnancy rate was higher in the first month compared to other months which is in accordance to this study. ${ }^{8}$

The increased infection rate was not there in this study neither in international studies and database review. ${ }^{7,8,10}$ This might be due to antibiotic coverage during and after the procedure. The drugs used in this study i.e. injection hydrocortisone and kanamycin have low drug reaction profile, are nontoxic and well tolerated.

Patients were informed about the possible risk of failure and empirical nature of treatment as exact place and success rate of the procedure in infertility cases is not yet well established but still many of the patients favored hydrotubation. In this study, the population belonged to low socioeconomic class and could not afford In vitro fertilization (IVF) and even intrauterine insemination (IUI). Several other studies have found that most women will choose hydrotubation because it is cheap, least - invasive and conception rate is increased without any increase in serious complications. $^{5,7,12}$

Immediate risk of hydrotubation included nausea vomiting moderate lower abdominal pain in majority of cases and mild peinumr vag bleeding in some cases. These side effects which are mostly due to pain cannot be minimized even with use of Lidocaine ${ }^{10}$. In patient of infertility, age is the main factor considered during treatment ${ }^{1}$ and in age above 35 years, time should not be wasted in procedures which are not yet recommended and standardized and have only empirical value. Hydrotubation if taken as an empirical treatment has also got a time saving factor as it will take only three months to decide whether hydrotubation will prove effective or not. The role of hydrotubation as a procedure requirement before IUI and IVF to increase its success rate. ${ }^{5,10}$

Safety of drugs used is another issue to be adderssed with. According to a review, tubal flushing with oil soluble contrast media increases conception rate. ${ }^{14}$ In other studies, results are significantly better with oil based contrast medium but it is costly and extravasation in peritoneal cavity can lead to anaphylactic reaction and lipogranuloma formation. An international study is under way to find out the effectiveness of hydrotubation in unexplained infertility with water soluble contrast medium but results are not yet available.

Further studies are required to find out most appropriate dosage of drugs used in hydrotubation and beneficial effect of addition of other drugs like progestogens in hydrotubation fluid.

\section{Conclusion}

In women with unexplained infertility, tubal hydration can increase conception rate and decrease the time to achieve pregnancy. In terms of higher pregnancy rate as compared to ovulation induction alone in unexplained infertility. The chances of conception decrease as more time is passed after hydrotubation. Right shoulder tip pain during the procedure is also positively correlated with pregnancy.

\section{References}

1. Mehmood G, Sadia S. Infertility Data of Maternal and Child Health Center, PIMS. J Surg Jun. 2001; 21-22: 10-3.

2. Edelstam G., Sjosten, A., BjurestenK,Ek L, Wanggren K. A new rapid and effective method for treatment of unexplained infertility. Hum Reprod. 2008; 23: 852856.

3. Wen H, Fu J, Tang H, Ge M, Feng L. Hydrotubation combined with Chinese herbal medicine for salpingitic infecundity: a systematic review and meta-analysis. Cell Biochem Biophys. 2015 Mar; 71 (2): 519-27.

4. Yapca OE, Delibas IB, Karaca Time-limited I, Kumtepe Y, Kadanali S. Time limited hydrotubation combined with clomiphene citrate treatment for unexplained infertility. Clin Exp Obstet Gynecol. 2015; 42 (3): 3114. PMID: 26152000.

5. Aboulghar M.A, Mansour R.T, Serour G.I, Amin Y, 
Abbas A.M. Ovarian superstimulation and intra-uterine insemination for the treatment of unexplained infertility. Fertil Steril. 1993; 60: 303-306.

6. Zou S.E, Jin Y, Ko Y.L, Zhu J. A new classification system for pregnancy prognosis of tubal factor infertility. Int J Clin Exp Med. 2014; 7: 1410-1416.

7. Adesiyun A.G, Cole B, Ogwuche P. Hydrotubation in the management of female infertility: outcome in low resource settings. East Afr Med J. 2009; 86: 31-36.

8. Giuliano E, Cagnazzo E, Bazzan E, Patella A, Marci R. Hysterosalpingo - contrast sonography: Is it possible to quantify the therapeutic effect of a diagnostic test? Clin Exp Reprod Med. 2012; 39 (4): 161-165.

9. Simsek E, Haydardedeoglu B, Hacivelioglu SO, Cok T, Parlakgumus A, Bagis T. Effect of cervical mucus aspiration before Intrauterine insemination. Int J Gynaecol Obstet. 2008; 103 (2): 136-9.

10. Walid A. Morad A, Ayman A. Abdelhamid. Prospective randomized study for hydrotubation with or without lidocaine before intrauterine insemination in unexplained infertility. Middle East Fertility Society Journal,
2012; 17: 250-255.

11. Aboulghar M.A, Mourad L.M, Al-Inany H.G, Mansour R.T. Prospective randomized study for hydrotubation Versus no hydrotubation before intra-uterine insemination in unexplained infertility. Reproductive Biomed. 2010; 20 (4): 543-546.

12. .Schippert C, Soergel P, Staboulidou I, Rocha G. The risk of ectopic pregnancy following tubal reconstructive microsurgery and assisted reproductive technology procedures. Archives of gynecology, 2011 Sep; 285 (3): 863-71.

13. Duffy JM, Johnson N, Ahmad G, Watson A. Postoperative procedures for improving fertility following pelvic reproductive surgery. Cochrane Database Syst Rev. 2009 Apr. 15; (2): CD001897. doi: 10.1002/14651858. CD001897.pub2. Review.

14. Mohiyiddeen L, Hardiman A, Fitzgerald C, Hughes E, Mol BW, Johnson N, Watson A. Tubal flushing for subfertility. Cochrane Database Syst Rev. 2015 May 1; (5): CD003718. doi: 10.1002/14651858.CD003718. pub4. Review. PMID: 25929235 